\title{
Strategy Analysis of Promoting Pollution Control of SMEs
}

\author{
Zhang Yanying ${ }^{1}$, Jiang Gui ${ }^{1 *}$, Yu Ziwei ${ }^{2}$ \\ ${ }^{1}$ Wuhan University of Science and Technology, Wuhan, 430065, China \\ ${ }^{2}$ Hubei Research Center for SMEs
}

\begin{abstract}
The pollution problem of SMEs is an important problem to be solved in the process of China's economic development. Based on the game theory, this paper takes the government and SMEs as the two sides of the game, constructing the game theory model of pollution control of SMEs, putting forward the strategies to promote the pollution control of SMEs according to the analysis results of the model.
\end{abstract}

\section{Introduction}

The report of the 19th National Congress of the Communist Party of China pointed out that the construction of ecological civilization is a great event for the development of the Chinese nation for thousands of years. As Xi Jinping said, lucid waters and lush mountains are invaluable assets. It is necessary to integrate the economic and social development and ecological civilization construction organically, so as to pursue good environmental benefits as well as good economic and social benefits.

According to the Ministry of Industry and Information Technology, by the end of 2018, the number of small and medium-sized enterprises (SMEs) in China has exceeded 30 million, accounting for $99 \%$ of the total number of enterprises. The booming SMEs play an important role in promoting the sustainable development of the national economy. However, at the same time, they also produce huge production resources consumption and environmental pollution problems during the production process. According to the investigation of the State Bureau of Statistics and the State Environmental Protection Administration, more than $80 \%$ of the industrial production has pollution problems, accounting for $60 \%$ of the pollution sources in China.

As far as the supervision of SMEs is concerned, the current regulatory measures are applicable to all enterprises, and they are also applied to the majority of SMEs at the same time. Due to the large number and wide distribution of SMEs and the huge cost of government supervision, the pollution control of SMEs in China has been very little. How to deal with the pollution problem of SMEs has become an important problem to be solved in the process of economic development in China.

In my opinion, the pollution control of SMEs is mainly a game between the government supervision department and the pollutant discharging enterprises. Therefore, this paper studies the game between them in the pollution supervision, trying to promote the possibility of government supervision and SMEs pollution control, and provide assistance for environmental protection.

\section{Analysis of pollution control of SMEs the perspective of game theory}

\subsection{Model hypothesis}

In this paper, we take SMEs and the government as the two sides of the pollution supervision game. SMEs in the market produce products at the same time discharge pollutants to the external environment. The government formulates pollution policies according to the bearing capacity of the environment.

Fistly, assuming that both sides of the game are completely rational economic men, their goals are to maximize their own interests. At the same time, assuming that the information is complete, both parties know the other party's choice of action, but the other party does not know the specific behavior.

Secondly, the strategic space of both players in the game is as follows: SMEs choose to control pollution or not; government (environmental regulatory agency) chooses to inspect or not inspect SMEs. Assuming that the probability of SMEs to control pollution is $x$, the probability of not treating pollution is $(1-x)$; the probability of government inspection is $y$, and the probability of not checking is $(1-y)$.

Thirdly, assuming that the regulatory cost required by the government is constant, that is, $K$. When SMEs register, they need to apply to the government for pollutant discharge permit and pay a license fee for this. Assuming that the amount of pollutant discharged by the enterprise is $n$ when applying for the license, the license fee for handling the permit is $M=a \cdot n(a>0)$, where $a$ is the rate of emission permit. Assuming that the actual pollution discharge of an enterprise is $n^{\prime}(n>0)$, in the case of pollution control, the enterprise needs to pay the cost of $M^{\prime}$ $=B>n(b>0)$, where $B$ is the cost rate of pollution control

\footnotetext{
* Corresponding author: 1012894113@qaq.com
} 
of the enterprise, and the government will give the enterprise a total of $T$ subsidies and tax incentives after checking the pollution control achievements of the enterprise. If the enterprise does not manage, it will cause adverse consequences to the environment. In order to make up for the damage of environmental resources, the government needs to pay this part of the governance cost, i.e. $M$. If the enterprise fails to pay the corresponding pollution control cost, but the government finds out the behavior of the enterprise due to inspection, it will impose a fine of $F=C \cdot n(c>0)$, where $C>>b$, the government will extract the part of $M^{\prime}=B>n(b>0)$ from the fine for pollution control.

\subsection{Model construction}

As mentioned above, the income function matrix of this model can be obtained, as shown in Table 1 .

Table 1. income matrix of SMEs and governments

\begin{tabular}{|c|c|c|}
\hline & pollute (x) & $\begin{array}{c}\text { Not pollute (1- } \\
\mathrm{x})\end{array}$ \\
\hline inspect (y) & $\begin{array}{c}\text { an-K-T, } \\
\text {-an-bn'+T }\end{array}$ & $\begin{array}{c}\text { an-K+cn'-bn', } \\
\text {-an-cn' }\end{array}$ \\
\hline not inspect (1-y) & an, -an-bn' & an-bn', -an \\
\hline
\end{tabular}

It can be seen from the above table that the expected returns of government departments are as follows:

$$
\begin{aligned}
& E 1=y\left[x(a n-K-T)+(1-x)\left(a n-K+c n^{\prime}-b n^{\prime}\right)\right] \\
& +(1-y)\left[x \cdot a n+(1-x)\left(a n-b n^{\prime}\right)\right]
\end{aligned}
$$

The expected returns of SMEs are as follows:

$$
\begin{aligned}
& E 2=x\left[y\left(-a n-b n^{\prime}+T\right)+(1-y)\left(-a n-b n^{\prime}\right)\right] \\
& +(1-x)\left[y \cdot\left(-a n-c n^{\prime}\right)+(1-y)(-a n)\right]
\end{aligned}
$$

As mentioned above, it is assumed that the government and SMEs are fully rational and pursue the maximization of their own interests. When deriving E1, let its reciprocal be zero, that is, if $\frac{\partial \mathrm{E} 1}{\partial y}=0$, formula (3) can be obtained.

$$
x=\frac{c n^{\prime}-K}{c n^{\prime}+T}=1-\frac{K+T}{c n^{\prime}+T}
$$

In the same way, if we take the derivative of E2 and make its reciprocal be zero, that is, if $\frac{\partial \mathrm{E} 2}{\partial x}=0$, we can get the formula (4).

$$
y=\frac{c n^{\prime}}{c n^{\prime}+T}=1-\frac{T}{c n^{\prime}+T}=\frac{c}{c+T / n}
$$

\subsection{Result analysis of game model}

According to formula (3), to improve the probability of government supervision, the following measures can be taken: 1) reducing the government inspection cost $K$; 2) reducing the pollution control reward $T$ paid by the government to enterprises; 3 ) increasing the fine rate $c$.

Rationality analysis:
When the income is fixed, the less the cost is, the easier the action subject will take the action. At the same time, when the cost is determined, the higher the income, the more willing the action subject is to take the action. For the government, the lower the inspection cost and the less rewards paid to the enterprises, the more likely the government is to act. Therefore, it is of positive significance to improve the inspection probability of the government, and measures 1) and 2) are reasonable. In the same way, increasing the penalty rate means the increase of revenue for the government, and can also improve the probability of government inspection, and the measures are reasonable.

According to formula (4), to improve the probability of pollution control of enterprises, there are the following situations: 1) increasing the fine rate $C ; 2$ ) increasing the government incentive subsidy $T$; 3) increasing the emission volume $n^{\prime}$.

Rationality analysis:

The penalty and reward measures adopted by the government are negative and positive incentives respectively. When the fine rate is increased, that is, when the negative incentive is enhanced, the cost of non pollution control SMEs will increase accordingly, which can promote the SMEs to avoid the action of no pollution control. When the government subsidies increase, that is to say, the positive incentive is enhanced. The enterprises can get more benefits from the pollution control behavior, which can prompt the SMEs to take pollution control actions. The conclusion 1) and 2) is reasonable. The cost of pollution control of enterprises is affected by many factors, such as the technical conditions of enterprises, the amount of pollutants discharged, the pollution treatment equipment and other factors. For a specific enterprise, the cost of the purchased sewage equipment is certain. When the maintenance cost is ignored, the larger the enterprise's emission volume is, the lower the marginal emission cost the enterprise will pay for each additional unit of pollution discharge. As the specified rate is fixed, in the case of not treating pollution, the larger the amount of pollution, one has to pay the higher the penalty cost. Therefore, the larger the discharge volume of the enterprise itself, the more likely the enterprise is to take pollution control action. The conclusion 3 ) is reasonable.

\section{Strategies to strengthen the supervision pollution control of SMEs}

\subsection{A normal inspection mechanism should be established to reduce the cost of government inspection}

The frequency of the opportunistic behavior of the SMEs in violating the regulations varies with the difficulty of supervision by the supervision department. When the supervision department does not supervise effectively, or the frequency of supervision is low, the probability of opportunism is high, and the probability of illegal discharge of pollutant discharging enterprises is high. If the monitoring frequency of supervision departments increases, the probability of enterprises' illegal emission 
detection will increase. Under the condition of fixed income, the higher the cost of opportunism, and the emission enterprises may take the initiative to reduce opportunistic behavior. SMEs have a large number and wide distribution, so their illegal discharge has the characteristics of "difficult to discover", "short of time", "difficult to obtain evidence".

Moreover, the life cycle of SMEs is relatively short, with an average of 7 years. In the process of supervision, the change and update of the enterprise list is also a big problem. The government can not be on duty to supervise the enterprises every day. Therefore, on the one hand, environmental supervision departments should improve their own monitoring technology level. On the other hand, they should pay attention to the use of scientific supervision methods, optimize the monitoring route, strengthen the links in the whole supervision work, and reduce the cost of information communication.

Firstly, in terms of the inspection mode, the working mode of regular inspection and reinspection after receiving reports is widely used in the traditional pollution control field. This work mode is too passive and easy for SMEs to form a routine to avoid inspection. The government can use the combination of regular inspection and random inspection to form a dynamic supervision on the pollutant emission of SMEs, so as to better grasp the pollution discharge situation of SMEs.

Secondly, we can reduce the cost by transferring the cost of government inspection to enterprises. SMEs should be required to install continuous monitoring devices and provide pollution treatment reports regularly when they apply for discharge permits. For those who refuse to install equipment, the government departments have the right to postpone the issuance of emission permits and refuse their production requests. For those who refuse to provide pollution control reports or provide false information in violating regulations in the process of regular inspection and random inspection, they shall be given corresponding administrative punishment.

Thirdly, we can selectively improve the inspection probability of some enterprises, especially those with severe pollution. Due to the lack of perfect pollutant emission reduction facilities for SMEs, there are often many heavy polluters. In order to alleviate the contradiction between the economic development of SMEs and the natural environment pollution, and to promote the development of circular economy, we should focus on the treatment of enterprises with heavy pollution. There are a lot of heavy pollution enterprises in the whole country. By focusing on improving the prevention and control concept of environmental pollution, it can play an environmental demonstration role for others. On the one hand, they can strengthen the recognition of the importance of environmental protection in the field of social and economic production through the treatment of key pollution enterprises, and on the other hand, they can apply key treatment to reflect the determination of pollution control, so as to lay the foundation for the future development of management work.

Fourthly, in the supervision of other social subjects, we can take public supervision to improve the detection probability of illegal enterprises. The number of enterprises that the government departments can pay attention to is limited, but the residents around the enterprises have a full understanding of the enterprises, and the community residents have the most say on whether the enterprises have related violations. The government can rely on the residents around the enterprise to transmit relevant information. Measures can be taken to let the residents actively supervise, such as increasing publicity intensity and frequency, so that residents can understand the importance of environmental protection. At the same time, measures should be taken to encourage reporting. If residents report illegal discharge of pollutants by SMEs, they will be given certain material rewards after investigated to be true.

\subsection{The cost of pollution control should be reduced; market mechanism should be introduced}

The reason why SMEs are not willing to carry out pollution control is mainly due to the high cost of pollution control, which is affected by the technical level and scale of SMEs. In terms of technical level, SMEs in China generally have low technical level and inferior production techniques, especially township enterprises often adopting technologies eliminated by urban industrial departments. Due to the low level of technology and the lack of human capital, SMEs not only pollute seriously, but also are difficult to reduce pollution through technological progress. In terms of enterprise scale, the economic scale of SMEs is too small. For example, according to national regulations, the economic operation scale of alkali recovery process, the key link in straw pulp papermaking wastewater treatment, is at least 1000 tons/day. There is a large gap between the emission scale of most SMEs and the above-mentioned economic operation scale, resulting in higher pollution treatment costs. From the perspective of enterprise pollution control facilities, the average life span of SMEs in China is 7 years, which is lower than the depreciation period of pollution control facilities, which also increases the cost of pollution control and reduces the willingness and ability of enterprises to control pollution.

The policy of "who pollutes, who governs" is applicable to enterprises with a certain scale. But it is not realistic for SMEs. Market mechanism can be adopted to solve the dilemma. As long as the government gives correct guidance, it is completely possible to concentrate SMEs in certain professional areas or industrial parks. Meanwhile, professional companies with environmental protection facilities operation qualification are responsible for centralized treatment, so as to solve the problem that pollution control facilities cannot meet the requirements of economic operation scale due to the small scale of enterprises.

On the basis of carrying out the pilot project of thirdparty environmental services, we can further promote the in-depth cooperation among environmental protection departments, industrial parks and enterprises, provide environmental protection contract management services for the government or enterprises through "environmental stewards", and charge fees according to the final pollution 
control effect or income. We should establish a service quality evaluation system, as well as supervision and restraint mechanism, and formulate supporting policies with clear responsibilities and orderly competition.it is necessary to carry out the operation of large-scale environmental protection facilities and pollution control services by a third party, or entrust environmental protection experts to monitor, supervise the construction and operation of environmental protection facilities, and other integrated services in the form of regions or groups.

\subsection{Information linkage should be strengthened; policy publicity and implementations should be promoted}

In the game hypothesis of this paper, the information between the government and SMEs is complete, but in reality, the information gap between the government and enterprises exists. Marked by the launch of the new environmental protection law in 2015, China's major industries and enterprises have ushered in the era of "cleaner production" since the central environmental supervision in 2016. Environmental protection storm roars past, but there are also many SMEs that are shut down because they do not understand the government policies. Therefore, the publicity and implementation of government policies is also very important for enterprises to choose pollution control.

Firstly, we should improve the service system of enterprises, take districts and counties as units, smooth the daily communication channels with enterprises, and establish regular contact and exchange mechanism. Many measures should be taken, such as supporting the industry to build private enterprise information service platform, guiding small and medium-sized enterprises practitioners to learn and understand national environmental protection policies, regulations, standards, as well as mastering the development trends of advanced green technology at home and abroad. We should strengthen intellectual support for green development of SMEs, and provide effective guidance and services for enterprises of different industries and sizes. Also, we should increase the training frequency of environmental protection laws and regulations, standards, technology and management knowledge of private enterprises, in order to help them to understand relevant laws and regulations.

Secondly, a platform should be built for government enterprise communication through a third party. Enterprises and the government can contact through environmental protection industry, society and other industrial organizations. They entrust associations and societies to participate in the formulation of environmental protection policies, plans and standards by purchasing services. Through industry organizations, the development demands of enterprises are fully expressed, and various policy requirements and information are timely transmitted to member enterprises to enhance their confidence and motivation in environmental governance. The government should keep track of the latest trend of the development of industry technology, publish economic and applicable technology and relevant information in real time, and help small and medium-sized enterprises to choose appropriate management technology or a thirdparty professional intermediary organization. At the same time, the third-party organizations should also give full play to the self-discipline role of industry organizations, regulate the behavior of members, and improve the quality of service.

\section{Conclusion}

SMEs are large in number and widely distributed. No matter how to strengthen supervision and reduce supervision cost, supervision organizations will always face the problem of cost. As a profit-making organization, the primary consideration of enterprises is their own interests. In the process of government enterprise game, the conflict of interests between the two sides will continue to exist. I believe that in order to make enterprises consciously control pollution and the government can effectively supervise, we can formulate strategies from the aspects of information communication between government and enterprise and the cost reduction of government and enterprise to promote the pollution control of SMEs.

\section{References}

1. Jin GR, Zhang L. China soft science, 1, 126-133, (2014)

2. Long WB, Li SH, Song XZ, Public Administration Review, 6, 25-56, (2015)

3. Long WB, Li SH, Xing DR. China Administration, 12, 66-73, (2017)

4. Wang GH, Innovation and Entrepreneurship Management, 2, 25-33 (2015)

5. Mei CM, Enterprise Economy, 12, 33-37, (2017)

6. Duan JJ. Charming China, 29, 39, (2017)

7. Zhou CG., Digital Users, 8, 222, (2018)

8. Chen Weiheng. Shenzhen Stock Exchange, 6, 59-64 (2018)

9. Guo T, Environmental Science and Management, 9, 10-14, (2019)

10. Chen H. Environment and Development, 4, 53, (2019) 